\title{
Laboreal
}

Volume $5 \mathrm{~N}^{\circ} 1$ | 2009

Ergonomia e desenvolvimento sustentável

\section{Ergonomía y desarrollo sustentable : conceptos y prácticas emergentes de experiencias cruzadas en Latinoamérica, Europa y África del Norte}

Ergonomia e desenvolvimento sustentável : conceitos e práticas emergentes de experiências cruzadas na América Latina, na Europa e no Norte de África Ergonomie et développement durable : concepts et pratiques émergentes d'expériences croisées en Amérique Latine, en Europe et dans le nord de l'Afrique Ergonomics and sustainable development : concepts and practices emerging from crossed experiences in Latin America, Europe and Northern Africa

\section{Cecília De La Garza e Mário Poy}

\section{OpenEdition}

\section{Edição electrónica}

URL: http://journals.openedition.org/laboreal/10149

DOI: $10.4000 /$ laboreal. 10149

ISSN: 1646-5237

\section{Editora}

Universidade do Porto

\section{Refêrencia eletrónica}

Cecília De La Garza e Mário Poy, « Ergonomía y desarrollo sustentable : conceptos y prácticas emergentes de experiencias cruzadas en Latinoamérica, Europa y África del Norte », Laboreal [Online], Volume $5 \mathrm{~N}^{0} 1$ | 2009, posto online no dia 01 julho 2009, consultado o 23 setembro 2020. URL : http:// journals.openedition.org/laboreal/10149; DOI : https://doi.org/10.4000/laboreal.10149

Este documento foi criado de forma automática no dia 23 setembro 2020.

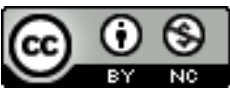

Laboreal está licenciado com uma Licença Creative Commons - Atribuição-NãoComercial 4.0 Internacional. 


\title{
Ergonomía y desarrollo sustentable : conceptos y prácticas emergentes de experiencias cruzadas en Latinoamérica, Europa y África del Norte
}

\author{
Ergonomia e desenvolvimento sustentável: conceitos e práticas emergentes de \\ experiências cruzadas na América Latina, na Europa e no Norte de África \\ Ergonomie et développement durable : concepts et pratiques émergentes \\ d'expériences croisées en Amérique Latine, en Europe et dans le nord de l'Afrique \\ Ergonomics and sustainable development : concepts and practices emerging \\ from crossed experiences in Latin America, Europe and Northern Africa
}

Cecília De La Garza e Mário Poy

1 Este número especial encuentra sus orígenes en una jornada organizada por la Sociedad de Ergonomía de Lengua Francesa (SELF Société d'Ergonomie de Langue Française) y la Unión Latinoamericana de Ergonomía ULAERGO durante el 2do. Congreso de ésta institución llevado a cabo en Bogotá, Colombia, en el mes de octubre del 2007.

El tema del congreso, "Ergonomía y competitividad: ¿Perspectivas o desafío ?" nos llevó a interrogarnos acerca de la noción de un sistema de "trabajo competitivo": ¿Cómo se define un sistema competitivo? ¿Cuáles son sus características? ¿Qué implicancias tiene en términos de las condiciones de trabajo y del bienestar de los trabajadores ? Y en lo que se refiere a un sistema de alto riesgo : ¿Qué implica la gestión de la seguridad y cuáles son sus relaciones con la competitividad?

3 Es así que, para intentar abordar estos interrogantes, la SELF y la ULAERGO decidieron organizar una jornada sobre el tema "Ergonomía, competitividad y desarrollo sostenible". El objetivo era entrecruzar puntos de vista entre ergónomos provenientes 
de diferentes realidades, latinoamericanos, europeos y africanos, respecto de las prácticas y los distintos campos de intervención, abordando el tema del desarrollo sostenible a través del análisis de la actividad de trabajo.

El resultado fructífero de esta jornada, introducida por David Caple, Presidente del IEA, nos animó a emprender una publicación a partir de las ponencias que fueron presentadas. La Revista Laboreal aceptó y apoyó constantemente este proyecto, y permitió que el mismo se concretizara.

5 El lector podrá constatar que los artículos reflejan una gran diversidad de puntos de vista, de prácticas, problemáticas y enfoques teóricos. En este sentido, la opción conceptual ha sido, deliberadamente, prestar testimonio de esta diversidad dado que, creemos y también lo deseamos, debería ser la base a partir de las cuales podamos fijarnos una agenda de intercambios y discusiones a fin de ampliar y profundizar el debate.

¿Cuál es la relación entre la ergonomía y el desarrollo sostenible ? Intentar responder a esta cuestión es precisamente nuestro desafío y es lo que vamos a mostrar en este número, partiendo de la definición del desarrollo sostenible.

7 El concepto se aplica al desarrollo socio-económico y fue formalizado por primera vez en el documento conocido como Informe Brundtland (1987), fruto de los trabajos de la Comisión Mundial de Medio Ambiente y Desarrollo de Naciones Unidas, creada en la Asamblea de las Naciones Unidas en 1983.

Dicha definición se asumiría en el Principio 3o de la Declaración de Río (1992) : "Satisfacer las necesidades de las generaciones presentes sin comprometer las posibilidades de las del futuro para atender sus propias necesidades".

9 El ámbito del desarrollo sostenible puede dividirse conceptualmente en tres aspectos: ambiental, económico y social (Cf. Figura 1). 
Figura 1 : Los 3 pilares de un desarrollo sostenible

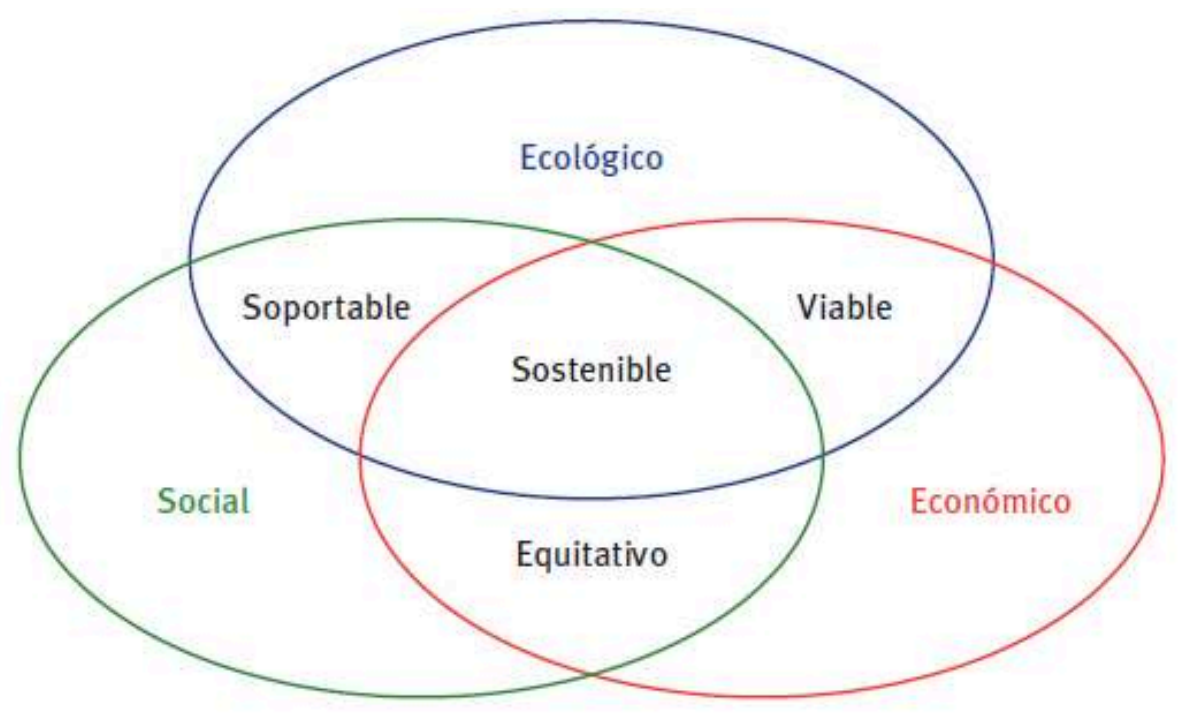

Figura 1: Los 3 pilares de un desarrollo sostenible

10 Se considera el aspecto social por la relación entre el bienestar social, el medio ambiente y la bonanza económica. Deben satisfacerse las necesidades básicas de la sociedad como son la alimentación, la vestimenta, la vivienda y el trabajo pues si la pobreza es habitual, el mundo estará encaminado a catástrofes de varios tipos, incluidas las ecológicas. Asimismo, el desarrollo y el bienestar social están limitados por el nivel tecnológico, los recursos y la capacidad del medio ambiente para absorber los efectos de la actividad humana. Ante esta situación, se plantea la posibilidad de mejorar la tecnología y la organización social de forma tal que el medio ambiente pueda recuperarse al mismo ritmo en que es afectado por la actividad humana.

Ahora bien, si aplicamos este mismo razonamiento al medio ambiente laboral podemos considerar que el desarrollo sostenible con relación a éste significa diseñar la tecnología (puestos y herramientas de trabajo) y la organización del trabajo de forma tal que los trabajadores y trabajadoras puedan no solo mantenerse en el trabajo, recuperarse de él sino, también, "construirse" (desarrollar nuevas competencias, trabajar en condiciones de bienestar mental y físico, ayudar a la construcción de la identidad como personas). De esta forma se contribuye al pilar social (mediante el bienestar del trabajador en su medio ambiente de trabajo y la prevención de accidentes de los sistemas de alto riesgo), al pilar económico (mayor eficacia y eficiencia de parte de trabajadores en buena salud, sin accidentes) y al ecológico (seguridad de los sistemas de alto riesgo).

Es en esta perspectiva, que los autores han dedicado en este número una reflexión especial acerca de la contribución de la ergonomía al desarrollo sostenible, a partir de sus propias experiencias, enfoques teóricos y prácticas en Europa, en América Latina y en África del Norte.

Con Elías Apud y Felipe Meyer (Chile) partiremos de los fundamentos teóricos y metodológicos de la ergonomía, para abordar después temas más recientes 
relacionados con la formación y los sistemas socio-técnicos resilientes, entre otros. Dichos autores nos dan un panorama del desarrollo de la ergonomía en Chile, bastante similar al de muchos países, a través de una serie de estudios realizados en empresas mineras de cobre en los últimos 10 años. Se tratan de evaluaciones descriptivas de puestos de trabajo tomando en cuenta las características físicas y fisiológicas de los trabajadores, en interacción con las actividades que estos realizan y el entorno físico (altura, frío). La noción de "puesto de trabajo" cubre aquí los accesos y circulaciones, las herramientas, el puesto de trabajo (asientos de los camiones). Los autores se interrogan ante la falta de perspectiva de futuro en el diseño de puestos de trabajo, lo cual significa une falta de anticipación, ignorando las características antropométricas de la población. Llegan así a afirmar la necesidad de diseñar empresas para "seres humanos" y muestran claramente como los problemas de salud y seguridad resultan de un diseño inadecuado y van, a largo plazo, totalmente en contra de un desarrollo sustentable de los trabajadores y de la empresa. Sin contar que la "corrección" de puestos de trabajo es mucho más cara y complicada que un diseño adecuado desde el principio. Sin embargo, un diseño adecuado y este es uno de los puntos más interesantes del artículo, no puede solo basarse en conocimientos antropométricos. Los autores expresan la necesidad de una visión sistémica del trabajo, integrando los conocimientos relativos a la antropometría como cognitivos, en interacción con las exigencias físicas y mentales del trabajo y, también, con el entorno físico y la variabilidad de la producción. El ejemplo de la minería nos deja ver como, si el diseño se limita al puesto de trabajo designado como el "camión", se olvida toda una parte del sistema socio-técnico, y en particular la tarea de "despacho" la cual implica une organización y una actividad comunicacional complejas.

14 Se concluye de este artículo que un diseño sustentable, cualquiera que sea el área, el tipo de empresa o de proceso debe asociarse con una visión sistémica del trabajo, y veremos que esto será confirmado por otros autores.

Si Apud y Meyer insisten en el diseño de empresas para "seres humanos", A. M. Seifert (Québec, Canadá) nos recuerda que los seres humanos - mujeres y hombres - no pueden ser estudiados de igual manera, puesto que no son tratados igual en las empresas. Seifert nos invita a mirar de manera distinta el trabajo de las mujeres y de los hombres si se quiere realmente contribuir a un desarrollo sostenible en el ámbito laboral. Según los países existen diferencias en los puestos y riesgos ocupados por mujeres, incluso cuando en la misma ocupación hay mujeres y hombres. La autora aborda entonces el desarrollo sostenible mediante el tema de conciliación "trabajo-familia", que desde luego no concierne solo a las mujeres, sino que también nos explica como se declina en el caso de las mujeres en relación con la flexibilización del trabajo, y por ende con el trabajo de tiempo parcial. La autora muestra a partir de 3 casos distintos (cajeras de banco, mucamas de hoteles y operadores telefónicos) realizados con el apoyo de los representantes sindicales, que este tipo de organización tiene un doble impacto negativo que va en contra de un desarrollo sostenible tanto de la empresa como de las trabajadoras. Por un lado se incrementan los riesgos sobre la salud física y mental, ya que se afectan el trabajo colectivo y las relaciones interpersonales, creando malestar, tensión, aumentando la carga de trabajo y, por otro lado, se crea ineficacia y pérdida de competencias. A través de sus estudios, la autora también insiste en el rol de las organizaciones sindicales como relevos y motores del cambio, tanto de las condiciones 
laborales, como de las representaciones del trabajo de las mujeres, motores del desarrollo sostenible de las condiciones laborales.

Siguiendo en la línea del bienestar/malestar en el trabajo, sobre todo mental, Laerte Sznelwar (Brasil) nos hace entrega de una serie de interesantes debates en torno al concepto de sustentabilidad. El autor pone en el centro de la reflexión el concepto mismo de trabajo para lo cual acude a los desarrollos conceptuales y metodológicos de la ergonomía de la actividad y de la psicodinámica del trabajo, enfoques ambos que, por defecto, abordan el problema del trabajo humando con un grado importante de minuciosidad. Sin embargo, como nos señala el autor, ambos enfoques guardan un grado de especificidad que les es propia : acoplamiento de los sistemas de trabajo a las características humanas en vista de promover la salud, la profesionalización y la producción, en el primer caso, y la reapropiación del sentido del trabajo en vistas de una reconstitución de la identidad y de la salud, en el segundo.

Con esta malla de análisis el autor nos presenta una intervención realizada en el marco de un Programa de Salud de Familia destinado a atender, prevenir y promover la salud, en tres ciudades importantes de Brasil, y cuyos equipos de trabajo están conformados por médicos, enfermeros, auxiliares de enfermaría y agentes comunitarios de salud. Los resultados de esta intervención a partir de ambos enfoques invitan a una reflexión respecto de la sustentabilidad en un doble sentido. Por un lado en el favorecimiento, por parte de los gestores y diseñadores del programa, de las condiciones que orienten la sustentabilidad del programa mismo, cuenta habida del tamaño, la cantidad y la diversidad poblacional de Brasil. Por otro lado, los resultados sugieren la necesidad de diseñar el trabajo mismo de los agentes de salud participantes, bajo criterios de sustentabilidad que permitan al mismo tiempo la promoción de la salud, el bienestar, la productividad y la identidad profesional. El autor introduce así la sustentabilidad en el medio de trabajo con relación a las condiciones laborales, a la acción realizada (el programa) tomando en cuenta el contexto en la cual se lleva a cabo.

En sus respectivos artículos, Marianne Lacomblez y Ricardo Vasconcelos (Portugal) y Vanina Mollo y Pierre Falzon (Francia) muestran cómo la formación profesional, basada en un conocimiento de la actividad real, concurre al desarrollo sostenible del propio individuo y, también, del medio de trabajo.

19 Marianne Lacomblez y Ricardo Vasconcelos, nos proponen una reflexión entorno a la problemática del desarrollo sustentable de la mano de las relaciones que entretejen el análisis ergonómico del trabajo y la formación profesional, y en particular la formación continua, declinadas bajo una triple perspectiva: la de la formación en el análisis ergonómico de la actividad de los agentes de prevención, el análisis del trabajo como herramienta para la formación profesional y, finalmente, la toma en cuenta y la valorización del recorrido profesional en el contexto de la transformación de los procesos productivos. En el recorrido que nos plantea, los autores nos recuerda que en los propósitos iniciales de la ergonomía, intentar conjugar la lógica de la salud, la seguridad y el bienestar con los de la eficacia productiva, ya se encontraban presente los desafíos impuestos por la sustentabilidad.

En su perspectiva diacrónica los autores evocan dos momentos clave en las relaciones entre el análisis ergonómico del trabajo y la formación profesional : la participación activa de los sindicatos que permite un encuentro fructífero entre los saberes "científicos" y los saberes construidos "en la acción", así como su valorización. Por otro lado, los cambios tecnológicos que imponen profundos cambios a la matriz productiva, 
impulsa a un replanteo de la formación profesional y que marca, entre otras cuestiones, el pasaje de la lógica de las "calificaciones" a la de las "competencias". Estas dos vías de reflexión llevan a plantear una tercera en la cual los autores afirman la necesidad de situar las acciones de formación dentro de los procesos de cambio que se suceden en los contextos productivos alertándonos, con justa razón, sobre las crecientes tendencias "psicologizantes" de situar todo el peso bajo la responsabilidad de los trabajadores.

Sus razonamientos, finalmente, nos invitas a reflexionar acerca del rol de los investigadores/formadores frente al trabajo, afirmando que sin un compromiso por un conocimiento detallado y fino del mismo (incluido la propia actividad del investigador), las cuestiones de la sustentabilidad solo serán una mera declaración de principios.

Vanina Mollo y Pierre Falzon introducen la noción de medio ambiente "capacitante" en el medio de trabajo con la idea subyacente de que el desarrollo sostenible es un proceso dinámico que se construye por medio de la actividad de los trabajadores. El desarrollo de competencias asiste a esa noción de capacitante teniendo un impacto tanto en la salud cognitiva, como en la fiabilidad de un sistema de alto riesgo (el hospital). Los autores muestran a través el análisis de un caso en un hospital, de un servicio de oncología, cómo se establece una organización eficaz mediante la construcción de un aprendizaje colectivo que tiene un doble objetivo: favorecer el desarrollo de conocimientos entre los médicos, y tomar la decisión más confiable y adecuada para tratar un caso. Sin embargo, favorecer dichas organizaciones tiene un costo ya que, por un lado, organizar reuniones colectivas, estructuradas alrededor de un tema específico, tomar una decisión colectiva después de toda una discusión, es algo que toma tiempo. Por otro lado, para que dicha organización tenga un papel de capacitante se necesitaría "rastrear" cada decisión, anotándola y justificando el pro y el contra con el fin de poder transmitir la información. Los autores concluyen que la idea de una organización capacitante seria sumamente útil, en particular en los sistemas de trabajo de alto riesgo, en donde las decisiones pueden tener un impacto fuerte en la seguridad de las personas o en la seguridad industrial.

Siguiendo en el eje de las organizaciones como factores contribuyentes al desarrollo sostenible de los trabajadores y de las empresas, Tahar-Hakim Benchekroun, Raouf Gham, Catherine Fournier, Francis Six, Mohamed Akrout (Francia y Túnez) reunieron sus conocimientos y experiencias para comentar 3 casos estudiados de empresas en Túnez. Los autores se basan en el enfoque de la Antropotecnologia propuesto por Wisner en los años 80 , aunque modificándolo al introducir el tema de la transferencia sostenible. La idea central es la introducción del desarrollo sostenible en la globalización. Se trata de un análisis de tipo "macro ergonomía" ya que se trataría de una toma de conciencia desde las políticas del gobierno (derecho laboral por ejemplo), de gestión de los recursos humanos y la organización de los procesos, hasta el diseño del puesto de trabajo. Si la visión sistémica del trabajo es necesaria para pensar, organizar y diseñar situaciones de trabajo sostenibles, los autores sostienen que el desarrollo sostenible en las empresas depende de las políticas del país. Y precisamente, los 3 casos expuestos - fabricación textil, industria de pañales y otros productos de bebés, y acondicionamiento de productos alimenticios - presentan características similares en cuanto a los problemas identificados. Dos de las empresas son europeas, o al menos en parte, y la tercera es tunecina. Sin embargo, ninguna respeta los horarios de trabajo, los periodos de vacaciones legales imponiendo, en todos los casos, ritmos y condiciones de trabajo extremadamente duros, problemas de mantenimiento de las 
máquinas, accidentes laborales, entre otros problemas. En realidad, lo que predomina es la productividad a pesar de la existencia de sistemas sumamente sofisticados en términos de calidad en una de ellas. Esto es lo que los autores describen como una modernización de geometría variable. Pero esta geometría parece agravarse a causa de la implantación de las empresas en otros contextos, al no promover la oportunidad de introducir un desarrollo sostenible en los países en vías de desarrollo. La ergonomía aquí se vuelve un instrumento "modesto" del desarrollo sostenible, contribuyendo al bienestar, a la salud y a la seguridad de los trabajadores, y por ende a la productividad y al desarrollo de la empresa.

Una reflexión sobre el desarrollo sostenible y el medio laboral no puede ignorar los lazos entre la organización del trabajo, productividad y seguridad del personal y seguridad industrial. Dos artículos abordan éste tema combinando la seguridad del personal y la seguridad industrial, asociando enfoques complementarios al análisis de la actividad de trabajo.

El artículo de José O. Gomes, Paulo V.R. Carvalho, David D. Woods, Tahar Hakim Benchekroum y Marcos R. S. Borges, resultado de una colaboración científica internacional (Brasil, USA, Francia), nos propone un abordaje de las relaciones entre seguridad y sustentabilidad, a partir de los desarrollos teóricos y metodológicos de la ingeniería de la resiliencia. En efecto, D. Woods, co-autor de éste artículo, es uno de los fundadores de este enfoque según él cual la gestión de los riesgos y de la seguridad depende de la capacidad de las organizaciones, grupos e individuos para anticipar cambios, fallas antes del evento crítico. En relación con los casos presentados aquí, la fiabilidad y por lo tanto la sustentabilidad de los sistemas de alto riesgo, con fuerte componente tecnológico, residen sobretodo en la capacidad de las personas y de la organización de poder capturar y tratar los microincidentes, las variaciones acontecidas, en vista de poder anticipar situaciones no deseadas.

A través del estudio de tres casos en sistemas socio técnicos complejos en Brasil una industria nuclear, un sistema de transporte aéreo (helicópteros) de una plataforma off shore y un centro de gestión de emergencias los autores nos ilustran sobre la cuestión. Utilizando múltiples métodos y técnicas de compilación de datos como la simulación de escenarios de accidentes mayores, incidentes técnicos, y técnicas de análisis cognitivo de tareas y de la actividad, los autores indagan sobre los elementos que hacen resilientes, o bien que fragilizan a dichos sistemas. En todos los casos, los resultados alcanzados sugieren que los puntos claves a tener en cuenta refieren a los procesos de coordinación de los equipos de trabajo, el diseño y la dinámica de las simulaciones, de los soportes de la comunicación llegando, incluso, al diseño de los puestos de trabajo.

Un sistema proactivo y sustentable, concluyen los autores, debe permitir utilizar medios eficaces para lograr un adecuado balance entre la seguridad y la alta productividad, así como poder anticipar los efectos de los cambios, en vista de poder prevenir las fallas potenciales. La resiliencia de los sistemas sociotécnicos de alto riesgo seria entonces una condición para mantener una sostenibilidad de las organizaciones y hasta del medio ambiente, contribuyendo a la fiabilidad de dichos sistemas. Sin embargo, los aportes de la ingeniería de la resiliencia a un enfoque sostenible no son tan claros y cabe preguntarse si se trata realmente de un enfoque novedoso, o si es una manera "novedosa" de nombrar viejas cuestiones. Aún así, nos parece interesante poner de relieve los puntos comunes entre los preceptos de la ingeniería de la resiliencia, usados por los autores y la noción de organización capacitante propuesta 
por Mollo y Falzon. El punto clave seria él del aprendizaje de las experiencias por las organizaciones. Se van así perfilando dos aspectos importantes de un enfoque sostenible en ergonomía : el diseño de puestos de trabajo y de las organizaciones, y la integración del aprendizaje de las experiencias a nivel individual, colectivo y organizacional. Veremos que, de cierta manera, el artículo de De la Garza y Poy confirma este punto.

Cecilia De la Garza (Francia) y Mario Poy (Argentina) presentan una doble reflexión aunando sus experiencias en dos contextos geográficos y socio económicos distintos, pero motivados por el mismo tema de investigación : la seguridad sostenible. Según los autores ocuparse de la seguridad, de un diseño seguro significa contribuir a una seguridad sostenible. Los autores presentan los resultados de dos casos poniendo énfasis por un lado en la seguridad de los trabajadores y, por el otro, en la seguridad industrial. En cierta medida, los enfoques teóricos y métodos de análisis pueden ser los mismos en ambos casos. La diferencia es sobre todo el momento en él cual la reflexión sobre la seguridad se lleva a cabo. En otros términos, una verdadera seguridad sostenible se refiere a una seguridad "proactiva" en relación con la producción del evento crítico (accidente, incidente). Dicha seguridad se basa en el conocimiento de los riesgos laborales reales mediante el análisis de la actividad, sin esperar la aparición del "accidente". Desde luego que el análisis de éste podrá contribuir a la seguridad, pero estaremos entonces en una seguridad de tipo "reactiva" para la cual existen una serie de modelos sistémicos y métodos de análisis de accidentes. El interés de este artículo es el de promover una prevención y una seguridad sustentables de las situaciones de trabajo, basadas en une dinámica "proactiva" mediante un diseño de tipo "human centred design", ecológico, de los puestos de trabajo, pero también de la organización, y en interacción con los riesgos inherentes propios a un puesto o a un proceso y con las capacidades de los trabajadores. Este último aspecto significa que la prevención sustentable implica una gestión de los recursos humanos en la cual los trabajadores y trabajadoras son considerados una fuerza real de la empresa, un capital que se protege.

Concluiremos con estas dos ideas que, de cierta forma, están presentes en todos los artículos como pilares clave de la sustentabilidad mediante la ergonomía : diseño (de puestos de trabajo, de la formación, de las organizaciones) y gestión de los recursos humanos basada en las características, capacidades y competencias de los actores del trabajo.

Los artículos que acabamos de presentar más que aportarnos conclusiones acabadas han sido, y son, un pretexto para el debate, para el intercambio de ideas y experiencias en torno a una problemática común. Esperamos, y deseamos una vez más, que este tipo de experiencias despierte nuevas inquietudes y contribuyan al afianzamiento y la profundización de los intercambios acá iniciados.

31 Asimismo quisiéramos resaltar y agradecer el apoyo sostenido de todos los autores, mediante sus valiosas contribuciones, a la revista Laboreal por su generosidad, y a David Caple, presidente del IEA, por su apoyo a este emprendimiento colectivo.

Finalmente, quisiéramos convocar a nuestros colegas y a todos aquellos interesados a continuar enriqueciendo estos intercambios inaugurados en 2007, y darnos cita formalmente en el próximo congreso de la IEA que tendrá lugar en Recife, Brasil, en 2012, aprovechando la reciente creación, por parte de dicha Asociación, del Comité Técnico sobre "Human Factors and Sustainable Development". 


\section{BIBLIOGRAFIA}

G. H. Brundtland (april, 1987). Report of the World Commission on Environment and Development. Nairobi.

\section{AUTORES}

\section{CECÍLIA DE LA GARZA}

Electricité de France, Département R\&D Management des Risques Industriels 1 Av. Général de Gaulle, 92140 Clamart, France

cecilia.de-la-garza@edf.fr

\section{MÁRIO POY}

Universidad de Palermo - Fundación Social Aplicada al Trabajo Geronimo Salguera, 1351 - 1177

Buenos Aires, Argentina

mpoy@fusat.org

mpoy@ciudad.com.ar 